subscapular artery, in this way retaining the opportunity for collateral circulation of the upper limb during cardiopulmonary bypass (Figure 2).

The cannula is fixed with the help of a tourniquet, and then this is stitched to the upper (lateral) edge of the wound with one stitch. The distal artery clamp is left in place up to the time of decannulation.

The cannula is removed at the end of the operation, and the brachial artery is restored with a 5-0 Prolene running suture (Ethicon, Inc, Somerville, NJ). In both presented cases, perfect perfusion during cardiopulmonary bypass could be provided.

\section{Discussion}

Cannulation of the axillary artery is counted as particularly advantageous for aortic dissection operations, and thus the anterograde flow into the dissected aorta is ensured.

The brachial artery cannulation applied by us in 2 cases (one on the right side and one on the left side) proved to be suitable and can be carried out quickly, and the cannulation site is at a good distance from the aortic arch. In this way there is less likelihood of postcannulation iatrogenic dissection spreading to the aorta. At the same time, the periscapular collateral circulation of the upper limb remained unaffected through the subscapular artery during cardiopulmonary bypass.

The brachial artery proved to be an easily accessible site of peripheral cannulation, which can be quickly carried out without complications.

In neither case were early or late neurologic complications observed in the upper limb.

\section{References}

1. Sabik JF, Lytle BW, McCarthy PM, Cosgrove DM. Axillary artery: an alternative site of arterial cannulation for patients with extensive aortic and peripheral vascular disease. J Thorac Cardiovasc Surg. 1995;109: 885-91.

2. Borst HG. Axillary artery for extracorporeal circulation [letter]. J Thorac Cardiovasc Surg. 1995;110:1775.

3. Neri E, Masseti M, Capannini G, Carone E, Tucci E, Diciolla F, et al. Axillary artery cannulation in type A aortic dissection operations. J Thorac Cardiovasc Surg. 1999;118:324-9.

4. Whitlark JD, Goldmann SM, Sutter FP. Axillary artery cannulation in acute ascending aortic dissections. Ann Thorac Surg. 2000;69:1127-8.

5. Miyatake T, Matsui Y, Suto Y, Imamura M, Shiiya N, Murashita T, et al. A case of intraoperative acute aortic dissection caused by cannulation into an axillary artery. J Cardiovasc Surg (Torino). 2001;42:809-11.

\title{
Atypical paraplegia after aortic intramural hematoma
}

\author{
Naotaka Motoyoshi, MD, ${ }^{\text {a }}$ Tsunehiro Komatsu, MD, ${ }^{a}$ Yoshimasa Moizumi, MD, ${ }^{a}$ and \\ Koichi Tabayashi, MD, ', Sendai, Japan
}

A ortic intramural hematoma (IMH) has previously been reported both at necropsy and in vivo and was recently described as a distinct entity. IMH appears to be characterized primarily by aortic wall hematoma without demonstrable intimal flap and occurs as a result of spontaneous rupture of the vasa vasorum ${ }^{1}$ or intimal fracture of an atherosclerotic plaque, which allows blood propagation into the aortic media. $^{2}$

On the basis of its pathologic feature, mesenteric ischemia by means of branch occlusion is supposed to be complicated with

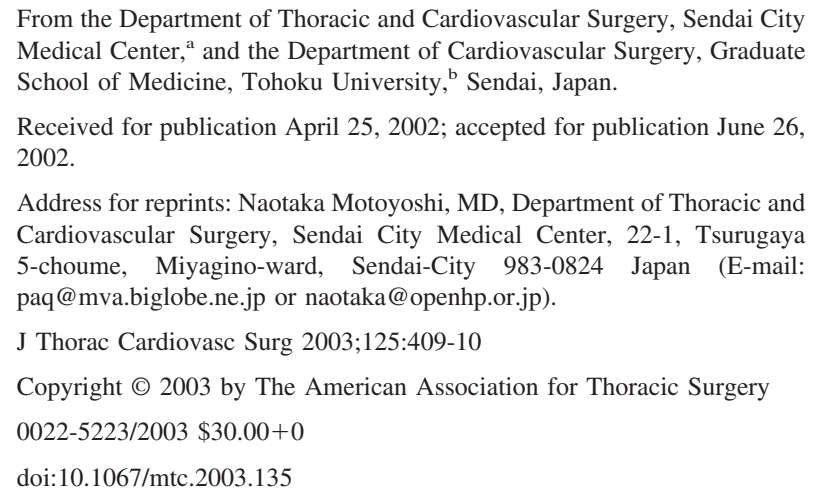

IMH. However, few incidents of perfusion disorder after IMH have been reported, whereas $2.5 \%$ to $12.0 \%$ of aortic dissections are complicated. ${ }^{3}$ We report a rare case of IMH resulting in ischemia of the spinal cord and the right iliopsoas muscle, representing atypical paraplegia.

\section{Clinical Summary}

At 5 PM on February 1, 2002, a 46-year-old patient had sudden onset of chest pain. On admission, he was hypertensive (182/80 $\mathrm{mm} \mathrm{Hg}$ ), and extremity pulses were palpated normally. Electrocardiography revealed a normal sinus rhythm and no ST-T-segment change. Computed tomography (CT) revealed IMH from the ascending to infrarenal abdominal aorta. Echocardiography showed no aortic regurgitation or pericardial effusion. Type A IMH with a 40-mm maximum diameter was diagnosed, and therefore antihypertensive therapy was preferred.

Weakness of the right thigh and calf was noted at 7 PM. After rapid, severe progress involving the left leg, the symptom was stabilized (modified Tarlov score of 0, Table 1). Sensory loss was observed on the right lower thigh. There was no other neurologic disorder. Cerebrospinal fluid (CSF) drainage at the lumber subarachnoid space was initiated at 10 PM. Primary CSF pressure was greater than $20 \mathrm{~cm} \mathrm{H}_{2} \mathrm{O}$. Rapid regression of the pressure to $10 \mathrm{~cm}$ $\mathrm{H}_{2} \mathrm{O}$ was marked after drainage. Two hours after drainage, the weakness was resolved, and within the subsequent 6 hours, the 


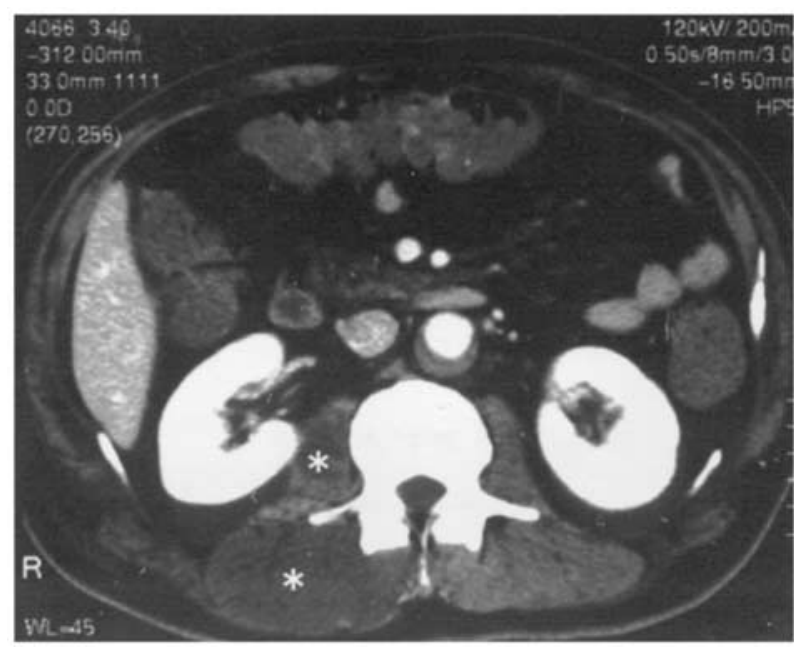

Figure 1. A low-density area was shown in the right iliopsoas muscle (asterisk), which represented lower CT number (ie, 35) than that of the opposite muscle area (CT number, 56).

\section{TABLE 1. Modified Tarlov scoring system}

\begin{tabular}{ll}
\hline 0 & No lower extremity motions \\
1 & Lower extremity motions without gravity \\
2 & Lower extremity motions against gravity \\
3 & Able to stand with assistance \\
4 & Able to walk with assistance \\
5 & Able to walk without assistance \\
\hline
\end{tabular}

neurologic status had improved to $3 / 5$ on the left and $2 / 5$ on the other. However, localized motor dysfunction on the right thigh remained even after drainage. An increase of serum creatine kinase ( $>20,000 \mathrm{IU})$ and myoglobinuria was detected. CT showed a lower-density area in the right iliopsoas muscle (Figure 1). After confirmation of ischemic rhabdomyolysis, mild hydration and diuretics were needed for renal protection. The enzyme level normalized within 35 days.

The drainage was discontinued after 6 days. The leg strength recovered to a $4 / 5$ grading on the left and a $3 / 5$ grading on the right. The subsequent course was unremarkable and was discharged 50 days later after neurologic improvement (score of 5) by 3 weeks of rehabilitation.

\section{Discussion}

Ischemic spinal cord injury after IMH has been reported previous1y. ${ }^{4,5}$ In our 104 cases of IMH, including types A and B, this was the first patient with spinal cord and muscle ischemia accompanied by IMH. It has become common that an urgent operation should be required for patients with type A IMH because dissection or rupture tends to develop in these patients. ${ }^{6}$ Others reported the maximum acute aortic diameter as either a risk factor for survival or a predictor for aortic enlargement in patients with IMH. ${ }^{7}$ In the present case the maximum diameter of disease was less than 45 $\mathrm{mm}$. No aortic regurgitation or pericardial effusion was detected. Therefore medical therapy was preferred. ${ }^{8}$ Finally, spontaneous regression of ascending aortic IMH was shown at 28 days after admission.

CSF drainage and its protective effect against ischemic spinal cord dysfunction after thoracoabdominal aortic surgery have been reported. ${ }^{9}$ Reduction of symptomatic paraparesis with IMH was achieved by means of CSF drainage in sporadic case reports. ${ }^{5}$ Moreover, we have performed 42 cases of CSF drainage as an adjunct with thoracoabdominal aortic replacement, and therefore we had no reason to hesitate with this drainage.

As in other reports, CSF pressure was primarily high in this patient. Also, pressure relief could regress symptomatic paraplegia immediately. The pressure might require ambient duration to be stabilized after ischemic insult without the drainage. Safi and colleagues ${ }^{9}$ proposed that a 3 -day course of the drainage was effective; however, the duration is not based on other reports. In our case, even at 5 days after drainage, the limb function became worse immediately after the pressure increase caused by unexpected kinking. The 3-day course is not absolutely practical. Prolongation of the course might be considered by patient. At 6 days, after limb function became stabilized on the on-off drainage test, the tube was removed.

Right hip joint flexion remained disordered, even after CSF drainage, in this patient. A high serum creatine kinase level and a low density within the right iliopsoas muscle on CT suggested ischemic rhabdomyolysis. This is the first reported case in which aortic IMH was able to occlude lumbar arteries branching out from the iliopsoas muscle. Care should be taken to determine partial involvement of atypical paraparesis in this type of case.

\section{References}

1. Gore I. Pathogenesis of dissecting aneurysm of the aorta. Arch Pathol Lab Med. 1952;53:142-53.

2. Murray CA, Edwards JE. Spontaneous laceration of the ascending aorta. Circulation. 1973;47:848-58.

3. Cambria RP, Brewster DC, Gertler J, Moncure AC, Gusberg R, Tilson $\mathrm{MD}$, et al. Vascular complications associated with spontaneous aortic dissection. J Vasc Surg. 1988;7:199-209.

4. Schaller B, Lyrer P. Anterior spinal artery syndrome: an important differential diagnosis of acute non-traumatic transverse spinal cord syndrome [in German]. Schweiz Rundsch Med Prax. 2001;90: 1420-7.

5. Killen DA, Weinstein CL, Reed WA. Reversal of spinal cord ischemia resulting from aortic dissection. J Thorac Cardiovasc Surg. 2000;119: 1049-52.

6. Maraj R, Rerkpattanapipat P, Jacobs LE, Makornwattana P, Kotler MN. Meta-analysis of 143 reported cases of aortic intramural hematoma. Am J Cardiol. 2000;86:664-8.

7. Kaji S, Nishigaki K, Akasaka T, Hozumi T, Takagi T, Kawamoto T, et al. Prediction of progression or regression of type A aortic intramural hematoma by computed tomography. Circulation. 1999;134: 495-507.

8. Moizumi Y, Komatsu T, Motoyoshi N, Tabayashi K. Management of patients with intramural hematoma involving the ascending aorta. J Thorac Cardiovasc Surg. 2002;124:918-25.

9. Safi HJ. How I do it: thoracoabdominal aortic aneurysm graft replacement. Cardiovasc Surg. 1999;7:607-13. 